\title{
A cell-free approach with a supporting biomaterial in the form of dispersed microspheres induces hyaline cartilage formation in a rabbit knee model
}

\author{
Javier Zurriaga Carda ${ }^{1,2}$ | Maria L. Lastra ${ }^{3}$ | Carmen M. Antolinos-Turpin ${ }^{4}$ | \\ Rosa M. Morales-Román ${ }^{4}$ | María Sancho-Tello ${ }^{1,5}$ | Sofía Perea-Ruiz ${ }^{4}$ | \\ Lara Milián $^{1,5}$ | Juan M. Fernández ${ }^{3}$ | Ana M. Cortizo ${ }^{3}$ | Carmen Carda ${ }^{1,5,6}$ | \\ Gloria Gallego-Ferrer $^{4,6}$ । José L. Gómez Ribelles ${ }^{4,6}$
}

\author{
${ }^{1}$ Departamento de Patología, Facultad de \\ Medicina y Odontología, Universitat de \\ València, Valencia, Spain \\ ${ }^{2}$ IMED (Innovación MÉDica), Hospital IMED, \\ Valencia, Spain \\ ${ }^{3}$ Laboratorio de Investigaciones en Osteopatías \\ y Metabolismo Mineral (LIOMM), Facultad de \\ Ciencias Exactas, Universidad Nacional de La \\ Plata 47 y 115 (1900), La Plata, Argentina \\ ${ }^{4}$ Center for Biomaterials and Tissue \\ Engineering (CBIT), Universitat Politècnica de \\ València, Valencia, Spain \\ ${ }^{5}$ INCLIVA Biomedical Research Institute, \\ Valencia, Spain \\ ${ }^{6}$ Biomedical Research Networking Center on \\ Bioengineering, Biomaterials and \\ Nanomedicine (CIBER-BBN), Valencia, Spain

\section{Correspondence} \\ José L. G. Ribelles, Center for Biomaterials and \\ Tissue Engineering (CBIT), Universitat \\ Politècnica de València, Valencia, Camino de \\ Vera s/n 46022 Valencia, Spain. \\ Email: jlgomez@ter.upv.es \\ Funding information \\ Comisión de Investigaciones Científicas de la \\ Provincia de Buenos Aires (CICPBA), \\ Universidad Nacional de La Plata, Grant/Award \\ Number: 11/X643; Agencia Estatal de \\ Investigación/Fondo Europeo de Desarrollo \\ Regional de la Unión Europea, Grant/Award \\ Number: MAT2016-76039-C4-1 2-R; Spanish \\ Ministry of Economy and Competitiveness \\ (MINECO)
}

\begin{abstract}
The objective of this study was to test a regenerative medicine strategy for the regeneration of articular cartilage. This approach combines microfracture of the subchondral bone with the implant at the site of the cartilage defect of a supporting biomaterial in the form of microspheres aimed at creating an adequate biomechanical environment for the differentiation of the mesenchymal stem cells that migrate from the bone marrow. The possible inflammatory response to these biomaterials was previously studied by means of the culture of RAW264.7 macrophages. The microspheres were implanted in a $3 \mathrm{~mm}$-diameter defect in the trochlea of the femoral condyle of New Zealand rabbits, covering them with a poly(L-lactic acid) (PLLA) membrane manufactured by electrospinning. Experimental groups included a group where exclusively PLLA microspheres were implanted, another group where a mixture of 50/50 microspheres of PLLA (hydrophobic and rigid) and others of chitosan (a hydrogel) were used, and a third group used as a control where no material was used and only the membrane was covering the defect. The histological characteristics of the regenerated tissue have been evaluated 3 months after the operation. We found that during the regeneration process the microspheres, and the membrane covering them, are displaced by the neoformed tissue in the regeneration space toward the subchondral bone region, leaving room for the formation of a tissue with the characteristics of hyaline cartilage.
\end{abstract}

\section{KEYWORDS}

articular cartilage regeneration, cartilage engineering, chitosan, microspheres, polylactide, rabbit knee model

\section{1 | INTRODUCTION}

Cartilage regeneration is a problem yet to be solved in clinical practice because it is a tissue with a low cell density and without vascularization. Osteochondral injuries often result in articular cartilage damage and premature osteoarthritis. This is already a huge problem, as it has been described to affect over $10.2 \%$ of adult population (Carmona, Ballina, \& Gabriel, 2001), and its effect is noted in a fivefold 
increase of articular prosthesis implanted in the period between 1994 and 2005 (Allepuz et al., 2014).

In clinical practice, a series of techniques have been applied for the regeneration or repair of articular cartilage that do not require the manipulation of cells outside the patient's organism, such as microfracture (Steadman, Rodkey, Briggs, \& Rodrigo, 1999) and mosaicplasty (Hangody et al., 1998). Other techniques based on tissue engineering require the implant of chondrocytes (autologous chondrocyte implantation, $\mathrm{ACl}$, or matrix-assisted chondrocyte implantation, MACl) (McCormick et al., 2014) or mesenchymal stem cells, MSCs, expanded ex vivo.

Cell-free approaches based on injuring subchondral bone to induce migration of pluripotent cells with chondrogenic capacity to the site of the defect are cheaper and technically easier to perform, but their intermediate and long-term results are poor, especially for younger and active patients (Goyal, Keyhani, Lee, \& Hui, 2013). It has been hypothesized that the implantation of a supporting biomaterial able to create the adequate biomechanical environment for the cells arriving to the regeneration site could significantly improve the quality of the new-formed cartilage. The implantation of cell-free scaffolds for cartilage regeneration greatly simplifies the treatment with respect to the implant of cell-laden scaffolds or hydrogels with lower mean costs and fewer surgeries needed, avoiding complications related to two-step techniques (Zhang, Cai, \& Lin, 2016). These scaffolds should be non-immunogenic and biodegradable to avoid deposits, porous to allow migration and adhesion of cells from subchondral bone, and mechanically stable to sustain the regeneration process. Bioresorbable polyesters such as polylactide (PLA) (Chu et al., 2016; Conoscenti et al., 2017) or polycaprolactone (PCL) (Martinez-Diaz et al., 2010; Vikingsson et al., 2015) have been used previously in cartilage engineering animal models. Cell-free strategies using PCL (Martinez-Diaz et al., 2010; Vikingsson et al., 2015) or biostable acrylic scaffolds of varying stiffness (Sancho-Tello et al., 2015; Sancho-Tello et al., 2017) probed in rabbit knee models their capacity to induce the formation of histologically high-quality tissue with the characteristics of hyaline cartilage. To increase the wettability of these hydrophobic materials, their combinations with hydrophilic coatings have been proposed (Lebourg et al., 2014).

In this work, we studied in a rabbit model the performance of a scaffolding material in the form of microspheres, combining stiff and hydrophobic microspheres made of a bioresorbable material such as PLLA with hydrophilic and compliant chitosan (CHT) microspheres. $\mathrm{CHT}$ has been proved to be a biodegradable and biocompatible material and has been proposed for cartilage regeneration. Although many works demonstrated good biocompatibility of CHT scaffolds (García Cruz et al., 2008; Lao, Tan, Wang, \& Gao, 2008; Lastra, Molinuevo, Blaszczyk-Lezak, Mijangos, \& Cortizo, 2018; Lastra, Molinuevo, Cortizo, \& Cortizo, 2017), others have reported increased inflammatory response of $\mathrm{CHT}$ materials when macrophages were studied (Almeida et al., 2014; Bitencourt Cda, Silva, Pereira, Gelfuso, \& Faccioli, 2015). This is why the possible inflammatory response was investigated using a model of RAW264.7 macrophages in culture in the presence of PLLA and/or CHT microspheres before implantation in animals.

\section{2 | MATERIALS AND METHODS}

\section{1 | Microspheres obtaining and characterization}

The PLLA used was of medical grade (Purasorb PL-18 by Corbion). PLLA microspheres were fabricated via an oil/water emulsion method. The oil phase consisted of $2 \% \mathrm{w} / \mathrm{v}$ PLLA solution in chloroform and the aqueous phase $4 \% \mathrm{w} / \mathrm{v}$ poly(vinyl alcohol) solution (Mw 130,000 Da, 99\% + hydrolyzed by Sigma Aldrich). Briefly, $20 \mathrm{ml}$ of PLLA solution was added dropwise via a syringe pump into $200 \mathrm{ml}$ of PVA solution under constant stirring at $750 \mathrm{rpm}$ at a feed rate of $1 \mathrm{ml} / \mathrm{min}$. An amount of $150 \mathrm{ml}$ of deionized water was added to assist with solvent evaporation, and the mixture was kept stirring for $24 \mathrm{hr}$. After stirring, the resulting suspension of microparticles was washed twice with water, filtered with ethanol via a $50-\mu \mathrm{m}$ filter, air dried, and vacuum dried prior to microparticles collection.

In order to improve the hydrophilicity, the microparticles were subjected to plasma treatment inside a Piccolo (Plasma Electronic) Microwave plasma chamber. The plasma treatment parameters were argon gas and $50 \mathrm{~Pa}$ initial gas pressure, with a gas flow rate of $160 \mathrm{sccm}$, for $600 \mathrm{~s}$.

An electrospun PLLA mat was prepared from a $2 \% \mathrm{w} / \mathrm{v}$ PLLA solution in a 30/70 mixture of $\mathrm{N}, \mathrm{N}$-dimethyl formamide and methylene chloride (Sigma) with a voltage of $25 \mathrm{kV}$ between the needle and the flat collector, a traveling distance of $15 \mathrm{~cm}$, a needle diameter of $0.5 \mathrm{~mm}$, and a feeding rate of $8 \mathrm{ml} / \mathrm{hr}$.

CHT microspheres were formed by neutralization of an acid solution of $\mathrm{CHT}$ in a basic solution subjected to an electric field. Medicalgrade CHT (Protasan UP B 80/20, Novamatrix) was dissolved at a concentration of $2.5 \% \mathrm{w} / \mathrm{v}$ in a $2 \% \mathrm{v} / \mathrm{v}$ acetic acid solution. The precipitating solution was prepared with $1 \mathrm{M} \mathrm{NaOH}, 0.5 \mathrm{M}$ $\mathrm{Na}_{2} \mathrm{SO}_{4} \cdot 10 \mathrm{H}_{2} \mathrm{O}$, and distilled water at a ratio $10 / 30 / 60$ and mixed with absolute ethanol in a 70/30 proportion to get better dispersion of the beads. The process consisted of passing the solution via a needle of $0.2-\mathrm{mm}$ inner diameter, placed at a height of $7 \mathrm{~cm}$ over the coagulant bath under agitation, at a constant feed ratio of $70 \mu \mathrm{l} / \mathrm{min}$ controlled by a syringe pump. A continuous electric field of $6 \mathrm{kV}$ was applied between the needle and the bottom of the coagulating bath, performing the process inside an Encapsulation Unit VARV1 (Nisco Engineering).

CHT microspheres were washed with distilled water several times until $\mathrm{pH} \approx 7$ by decantation and aspiration of the supernatant.

Confined compression tests were performed in a Microtest Electromecánica SCM3000 95 instrument. Prior to the test, the microspheres were kept immersed in liquid water for $24 \mathrm{hr}$. The microspheres were introduced into a plunger cylinder device, made of poly(tetrafluoroethylene) (PTFE), with a diameter of $5 \mathrm{~mm}$. The initial thickness of the microsphere layer was $1 \mathrm{~mm}$. The cylinder had perforations in the lower part that allow eliminating the water that comes out of the microspheres during the compression test. A deformation 
ramp was applied at a speed of $1 \mathrm{~mm} / \mathrm{min}$ until the force measured in the load cell was $10 \mathrm{~N}$. The result expressed in terms of a compression module, $K$, is the average of five measurements of a confined cylindrical sample of cross section $S$ and initial height $I_{0}$

$$
K=\frac{F / s}{\Delta I / \Lambda_{0}},
$$

where $F$ is the applied compression force and $\Delta l$ is the measured deformation.

The morphology of PLLA microparticles was observed by field emission scanning electron microscopy (FESEM) (Ultra 55, Zeiss Auriga Compact, Germany). The images were taken at $1 \mathrm{kV}$ using platinum-coated samples (JFC 1100, JEOL, Japan device). Images of the $\mathrm{CHT}$ microspheres, swollen in water, were obtained by binocular loupe (MZ APO, Leica Microsystems, Germany).

\section{2 | Raw264.7 culture and incubations: evaluation of cytotoxicity}

Cell viability and the eventual cytotoxicity of the CHT, PLLA and PLLA $+\mathrm{CHT}$ microspheres (at a 1:1 volume ratio) were studied in vitro using murine RAW264.7 macrophage. This cell line constitutes an excellent model for studies of cytotoxicity of different substances on biological systems, because RAW264.7 expresses different markers of cellular activity such as interleukin synthesis, nitric oxide (NO) production, and expression of nitric oxide synthases (NOS) against toxic substances (Denlinger et al., 1996). These cells were maintained in Dulbecco's modified Eagle's medium (DMEM) without phenol red supplemented with $10 \%$ fetal bovine serum (FBS) and antibiotics (100 U/ml penicillin and $100 \mathrm{~g} / \mathrm{ml}$ streptomycin) at $37^{\circ} \mathrm{C}$ in a $5 \% \mathrm{CO}_{2}$ atmosphere. The polymeric microspheres were sterilized with ethanol $(70 \% \mathrm{v} / \mathrm{v})$ overnight. Then, they were washed three times with Milli-Q sterile water and were incubated with DMEM without phenol red, $10 \% \mathrm{FBS}$, and antibiotics overnight at $4^{\circ} \mathrm{C}$ before its use. Cell viability was evaluated by the 3-(4,5-dimethylthiazol-2-yl)2,5-diphenyl tetrazolium bromide (MTT) assay (Lastra et al., 2017). Briefly, $1 \times 10^{5}$ cells/tube were seeded inside $500 \mu$ l Eppendorf tubes with the microspheres and cultured for 24 or $48 \mathrm{hr}$. After these culture periods, the cells were incubated for two additional hours with a solution of $0.1 \mathrm{mg} / \mathrm{ml}$ MTT (Sigma). After washing, the formazan precipitate was dissolved in $100 \mu$ of dimethyl sulfoxide and the absorbance reading was measured at $570 \mathrm{~nm}$ using an automatic ELISA plate reader (Infinite ${ }^{\circledR}$ F50, Tecan Trading AG, Switzerland). Results were expressed as \% basal, taking as basal the cell viability under control conditions at $24 \mathrm{hr}$. The potential cytotoxicity of the microspheres was evaluated by the interleukin-1 $\beta$ (IL-1 $\beta$ ) and the NO production released into the culture medium by RAW264.7 cells after 24 and $48 \mathrm{hr}$. IL-1 $\beta$ was measured by ELISA kits (BD OptEIA mouse IL-1 $\beta$ ELISA) following the manufacturer's recommendations. The stable end-product of NO production was assessed using the Griess reagent (Fernández, Cortizo, \& Cortizo, 2014). Briefly, $200 \mu$ l samples of conditioned media or nitrite standards $(0-100 \mathrm{nM})$ were mixed with
$200 \mu \mathrm{l}$ of Griess reagent ( $1 \%$ sulfanylamide and $0.1 \%$ naphthylethylenediamine in $5 \%$ phosphoric acid) and absorbance was measured at $530 \mathrm{~nm}$.

Results are expressed as the mean $\pm S E M$ and were obtained from two separate experiments performed in quadruplicate. Differences between groups were assessed by one-way analysis of variance (ANOVA) with Tukey's post hoc test. For non-normal distributed data, nonparametrical Kruskal-Wallis with Dunn's post hoc test was performed using GraphPad InStat v. 3.00 (Graph Pad Software, San Diego, CA). $p<.05$ was considered significant for all statistical analyses.

\section{3 | Rabbit model}

In order to evaluate cartilage regeneration, an animal model was designed following the International Cartilage Repair Society (ICRS) guide (Hoemann et al., 2011). With the approval of the ethics committee of the Universitat de València, 12-week-old male New Zealand rabbits weighing about $1800 \mathrm{~g}$ were used. The surgical technique included a medial parapatellar approach to the right knee, eversion of the patella, and the creation of the osteochondral injury in the femoral trochlear groove using a 3-mm-diameter punch. The cartilage defect was filled with wet biomaterial microspheres (with the consistency of a paste), and over them a circular membrane composed of PLLA, of $3 \mathrm{~mm}$ diameter and $100 \mu \mathrm{m}$ thick, was placed. This membrane, with the clot formed by the subchondral bone injury bleeding, ensured mechanical stability without the need for sutures or fibrin glue.

A total of 9 rabbits were used for each experimental group to have results statistically significant. In order to limit the number of experimental animals, only two microsphere formulations were considered: In one of the series, named PLLA, only PLLA microspheres were implanted, in the second series, named PLLA+CHT, a mixture of PLLA and $\mathrm{CHT}$ (in a 1:1 volume ratio) was implanted. A different group named $M$ was used to evaluate the biological ability of the animals to repair the injury, where the cartilage defect was created including the injury of the subchondral bone, but only the PLLA membrane was placed. The control group of native cartilage consisted of the left knees of the rabbits. It must be noted that two rabbits deceased during the initial anesthetic part of the surgery earlier to the implantation of the scaffolds.

12 weeks after the procedure, the animals were sacrificed using a veterinary-controlled thiopental overdose. The knees were extracted and the macroscopic evaluation was carried out following the ICRS proposal (Van den Borne et al., 2007). They were then processed, including a fixation in $10 \%$ buffered formaldehyde for 5 days followed by decalcification using Osteosoft ${ }^{\circledR}$ for 5 weeks. The samples were then included in polyester wax for microscopy, with low melting point, and 5-7- $\mu \mathrm{m}$ seriated sections were performed. The different sections were stained using hematoxylin-eosin (H\&E), toluidine blue, and Masson trichromic for the different microscopic measurements. Polarized light microscopy was also used in order to observe the orientation of the collagen fibers. 
Microscopic evaluation was also performed following the ICRS II scale (Mainil-Varlet et al., 2010), where each parameter is scored using a 100-unit visual analogue scale, with a score of 0 being assigned for the properties considered indicative of fibrous cartilage or poor quality articular hyaline cartilage, and 100 for good quality articular hyaline cartilage. However, we added three parameters to evaluate the presence of non-reabsorbed membrane and microspheres, as well as inflammation in the areas where they were observed. The quality of the regenerated cartilage obtained was also evaluated using the ImagePro Plus 7.0 program with morphometric criteria: we measured cartilage and subchondral bone thickness, cell density, interdigitation index (the index obtained by dividing the length of cartilage surface measured in a particular sample and the ideal curved surface a normal cartilage would have), the number of non-reabsorbed microspheres and their depth, area of non-reabsorbed membrane, and its depth, and, in the cases where they were observed, the area and depth of cyst formation or inflammation.

Two independent observers carried out all the measurements, and $\kappa$ coefficients (Landis \& Koch, 1977) were calculated in order to establish the consistency of the observations.

The statistical analysis included a Kolmogorov-Smirnov test to determine the normal distribution of the data, an R-Pearson test to establish the absence of correlation between variables, an ANOVA test for mean comparison, a $\chi^{2}$ test for categorical variables and a Scheffe or Games-Howell test for multiple mean comparisons depending on whether the variances were homogeneous or not. Significant differences were established below $p<.05$.

\section{3 | RESULTS}

\section{1 | Supporting biomaterials}

Figure 1 shows the morphology of the PLLA and CHT microspheres. The diameter was $40 \pm 20 \mu \mathrm{m}$ for PLLA and $205 \pm 5 \mu \mathrm{m}$ for CHT. The combination of the rigid and hydrophobic microspheres of PLLA with the hydrophilic ones of $\mathrm{CHT}$ allows modulating the biomechanical environment found by the cells that invade the site of the cartilage defect. The mechanically confined compression test aims to simulate the situation that occurs in vivo, with the implanted microspheres in the cartilage defect. In this test, the microspheres are introduced into the PTFE cylinder (inset in Figure 2), to a thickness of $1 \mathrm{~mm}$, and distilled water is added to fill the space between the microspheres. The stress-strain diagram (Figure 2) of the test carried out with $\mathrm{CHT}$ microspheres shows how, at the beginning, with a tension close to 0 , the piston moves, eliminating the excess water (I). In a second stage (II), the applied force grows when the microspheres contact each other and start to deform, and it is Stage II that has been considered representative of the elastic modulus during the regeneration process. The origin of deformation was detected by the shift of the stressstrain curve from the straight line fitted to the experimental data in Stage I. When the microspheres occupy all the volume, the tension grows rapidly representing the elastic modulus of the block material (III). The value of the compression module was calculated in the strain

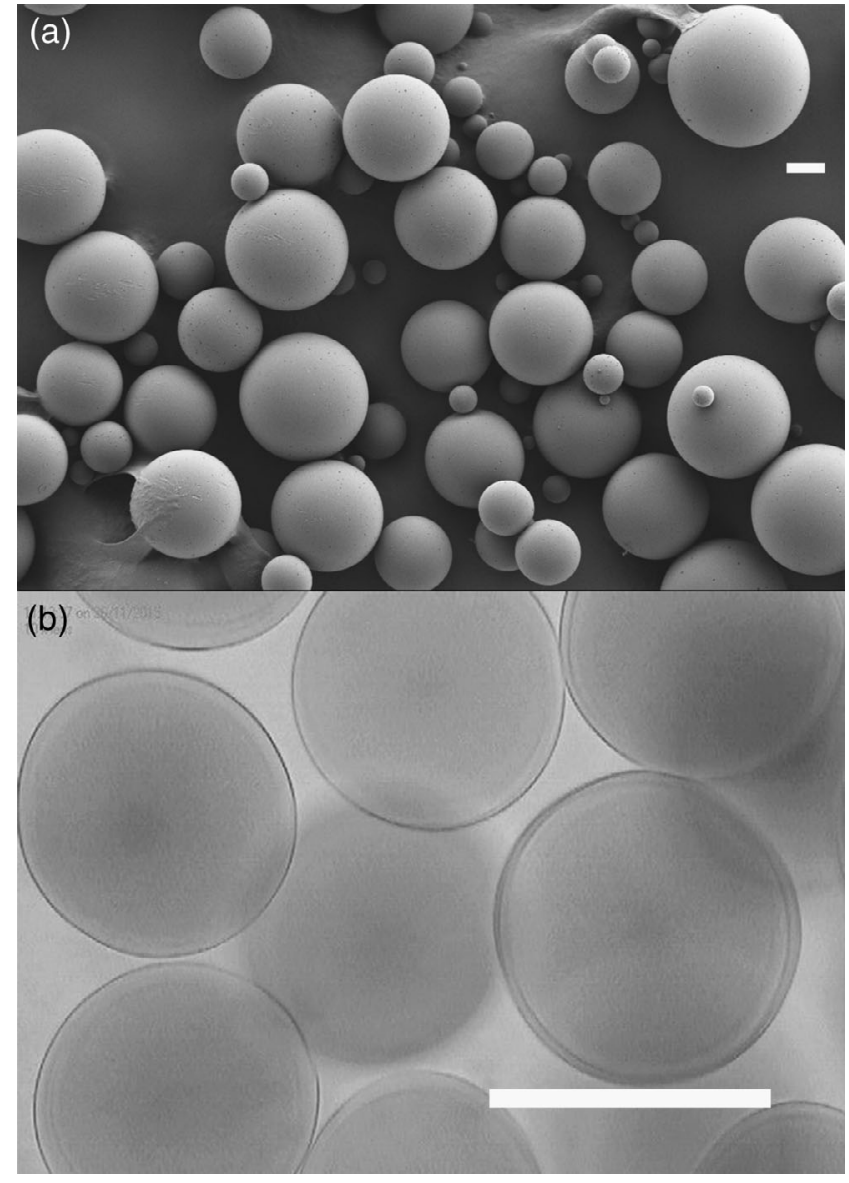

FIGURE 1 (a) FESEM picture of PLLA microspheres produced by an oil/water emulsion (dimension bar $20 \mu \mathrm{m}$, the diameter of these microspheres was $40 \pm 20 \mu \mathrm{m}$, mean $\pm S D$ ); (b) binocular loupe pictures of $\mathrm{CHT}$ microspheres produced by neutralization of the $\mathrm{CHT}$ acidic solution swollen in water (dimension bar $200 \mu \mathrm{m}$, the diameter of these microspheres was $205 \pm 5 \mu \mathrm{m}$, mean $\pm S D$ ). CHT, chitosan; FESEM, field emission scanning electron microscopy; PLLA, poly(Llactic acid)

interval between 0.1 and 0.2 that corresponds to the physiological deformation of cartilage. The values obtained were $1.2 \pm 0.25 \mathrm{MPa}$ in the case of PLLA, $0.64 \pm 0.03 \mathrm{MPa}$ for the mixture of PLLA and CHT microspheres, and $0.21 \pm 0.08 \mathrm{MPa}$ for $\mathrm{CHT}$.

\section{2 | In vitro tests and induction of inflammation mediators}

As a first step, before implantation of the biomaterial in the animals, inflammatory response was tested in vitro. We evaluated the cell viability of RAW264.7 macrophages with the different microspheres compared with spheres-free culture (control conditions). Figure 3 shows no significant differences in viability of cells cultured in the presence of different microspheres and the control condition, after 24 or $48 \mathrm{hr}$ of incubation. The influence of microspheres on the inflammatory response was evaluated by measuring the pro-inflammatory cytokines $\mathrm{IL}-1 \beta$ and the NO production by macrophages in culture. We found no 


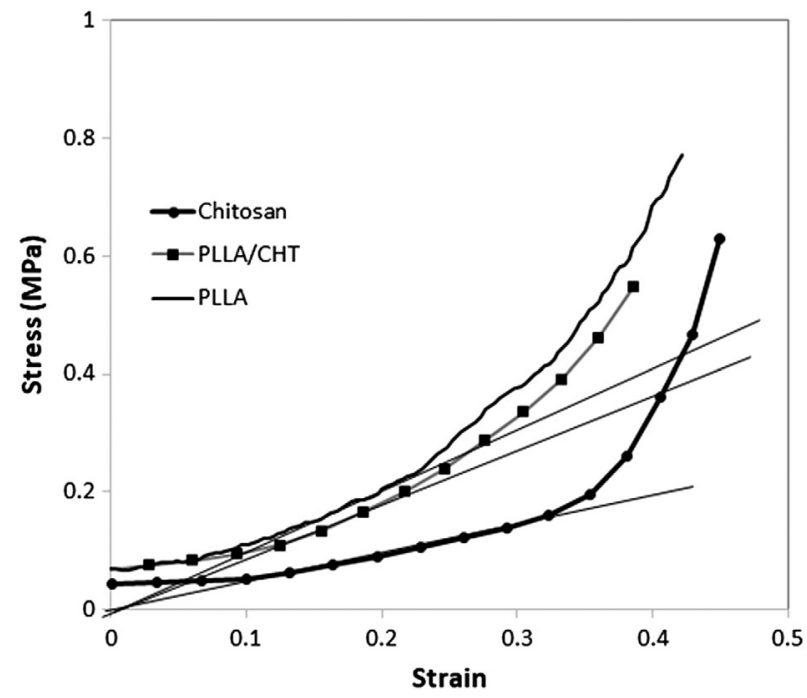

FIGURE 2 Stress-strain compression test of the wet microspheres confined in a plunger cylinder device (see the text). $(\bullet)$ Chitosan, CHT; (-) poly(L-lactic acid), PLLA; and (ロ) PLLA+CHT

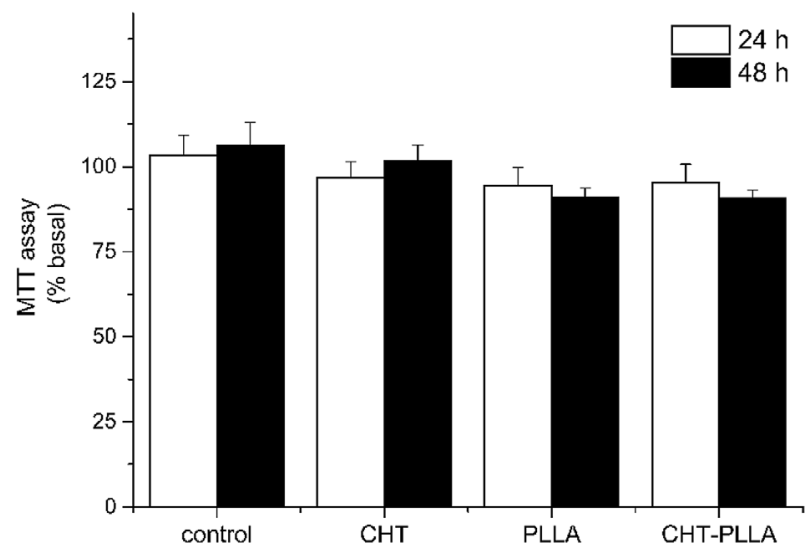

FIGURE 3 MTT assay results expressed as \% basal, taking as basal the cell viability under control conditions at $24 \mathrm{hr}$. RAW 264.7 macrophages cultured for 24 and $48 \mathrm{hr}$ with chitosan, CHT; poly(L-lactic acid), PLLA; and $\mathrm{CHT}+$ PLLA microspheres. Data represent the mean \pm SEM

differences in IL-1 $\beta$ produced after the cells were grown in the presence or absence (control conditions) of microspheres after 24 or $48 \mathrm{hr}$ (Figure 4a). Similar results were obtained for NO produced (Figure 4b), although we only found a significant increase in NO production after 48-hr incubation of cells grown in the presence of PLLA+CHT compared with the control (Figure $4 b, p<.05$ ).

\subsection{Rabbit knee model}

No inflammatory reaction or infection was observed throughout the postoperative period, and all of the animals were found to have a normal walking behavior and were able to maintain bipedalism within 3 weeks after the procedure.

Figure 5 shows representative histology images of the regenerating zone 3-month postimplantation of only PLLA microspheres
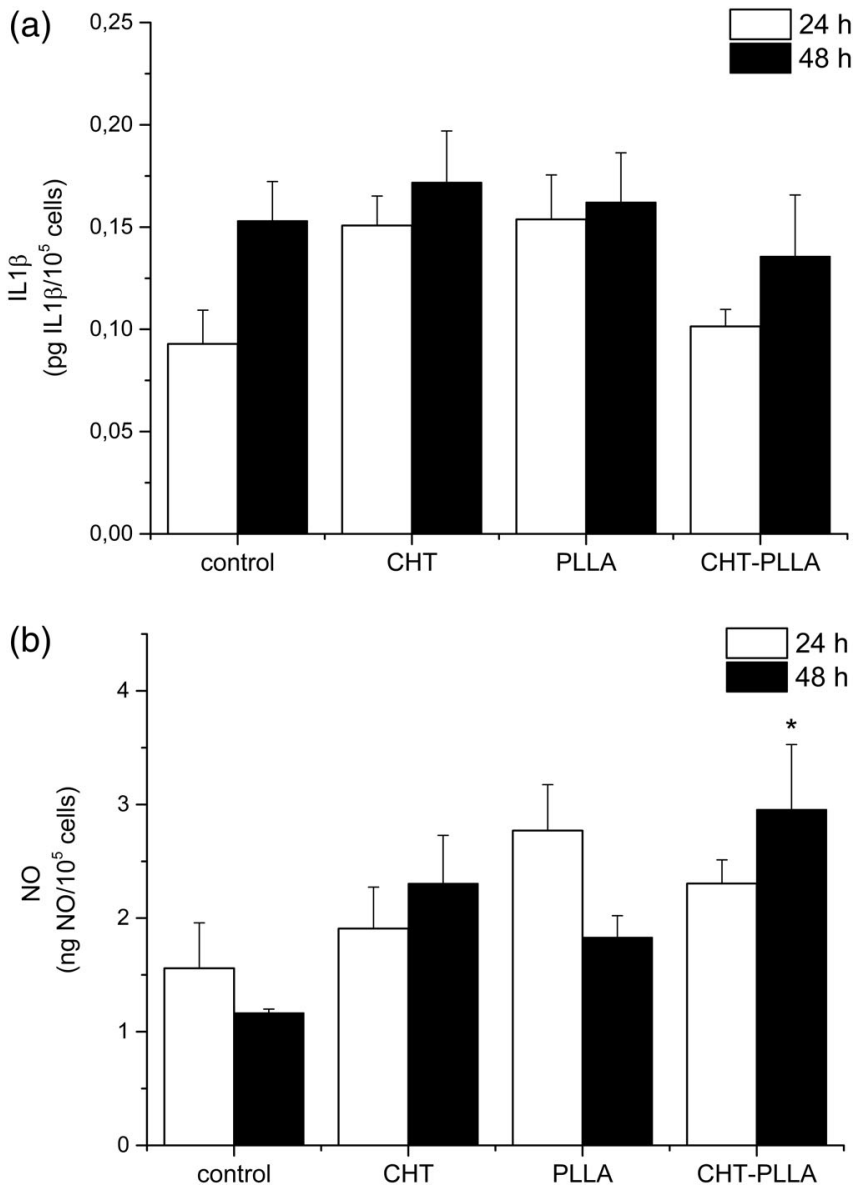

FIG URE 4 Cytotoxicity assay. Production of interleukin-1 $\beta$ (IL-1 $\beta$ ) (a) and NO (b) by RAW 264.7 macrophages upon incubation with different microspheres. Data represent the mean \pm SEM. $* p<.05$ versus control. NO, nitric oxide

(Figure 5a) or a mixture of PLLA and CHT microspheres (Figure 5c), in both cases covered by a PLLA membrane. The histology of the newly formed tissue when only the membrane was implanted after osteochondral injury is shown in Figure $5 \mathrm{~d}$. It is worth noting that the degradation time of PLLA is much longer than 3 months; thus, both the PLLA membrane and PLLA microspheres are expected to be in the regeneration zone yet. Interestingly enough both are shifted out of the cartilage zone, as observed in Figure 5a (microspheres are indicated by the yellow arrows) and at a higher magnification in Figure $5 \mathrm{~b}$, while the rest of the membrane are shown by the green arrows. The number of particles shown in the histology when a 50/50 mixture of PLLA and CHT microspheres is implanted is much smaller than when only PLLA microspheres are implanted, and they appear at a larger distance to the articular surface. A macroscopic view of the articular surface 3-months postimplantation is shown in Figure 6. The areas of regenerated cartilage in PLLA series had a smooth whitecolored surface, with only occasional irregularities or small lumps (Figure 6a). Cross section also shows a quite homogeneous cartilage layer in the regeneration site (Figure 6b). Nevertheless, when PLLA and $\mathrm{CHT}$ microspheres were implanted, irregularities, in the form of lumps, at the surface were more frequent, appearing even at some 

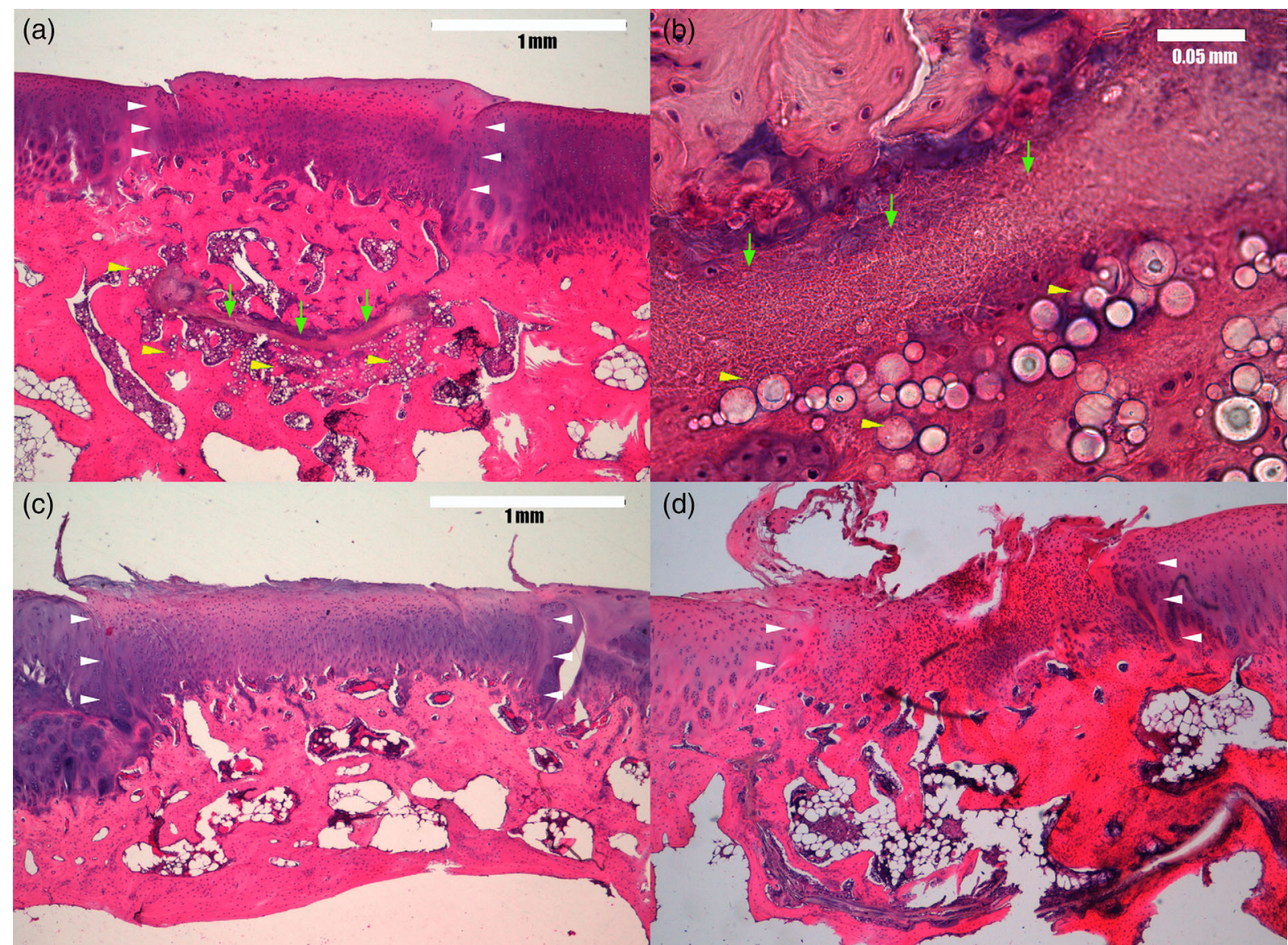

(d)

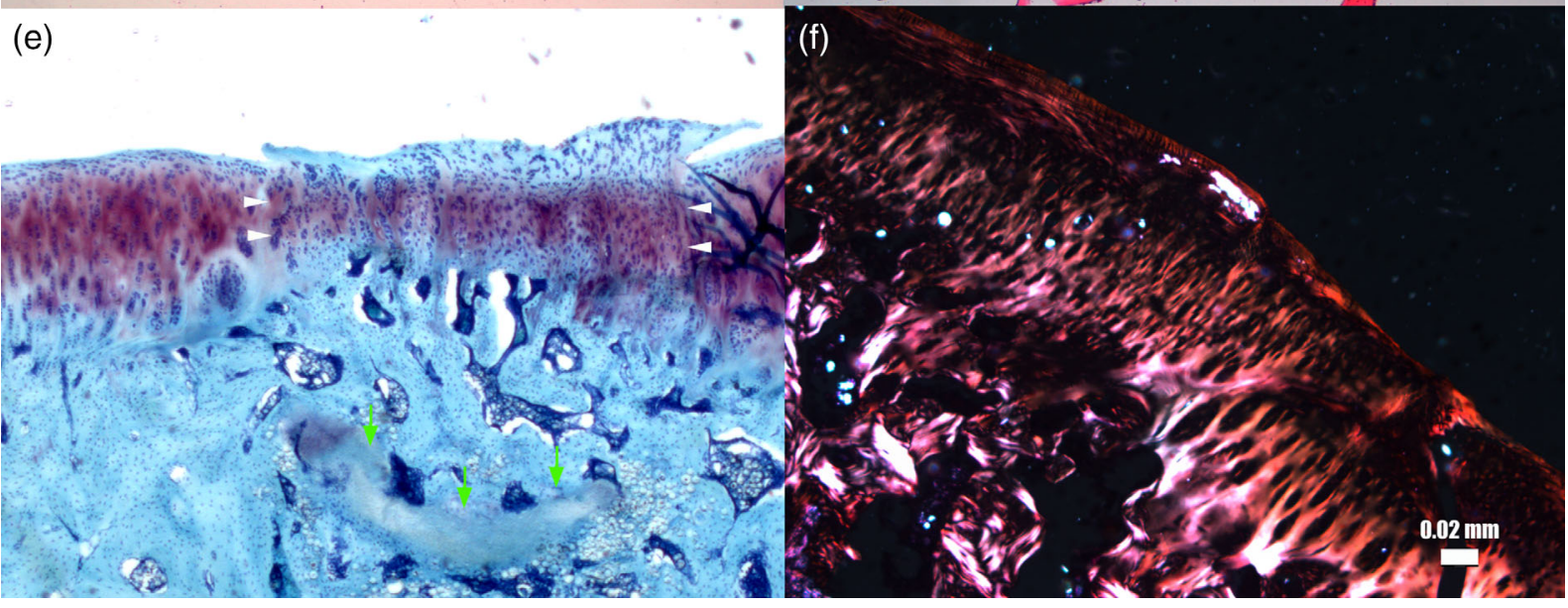

FIGURE 5 Microscopic views of the experimental areas for groups (a) PLLA (H\&E), (b) PLLA sample with a higher magnification showing the remaining microspheres and membranes located in subchondral bone (H\&E), (c) PLLA + chitosan, PLLA + CHT (H\&E), (d) series M (H\&E), (e) sample of group PLLA + CHT stained with toluidine blue in order to evaluate metachromasia, and (f) image viewed under polarized light microscopy of a sample of the PLLA group. White arrows show the limit of the practiced cartilage defect, and yellow arrows and green arrows indicate some of the PLLA microspheres and the membrane remaining after 3-month implantation. Individual scores in the ICRS II scale for each category and group are summed up in Table 2. CHT, chitosan; H\&E, hematoxylin-eosin; ICRS, International Cartilage Repair Society; PLLA, poly(L-lactic acid)

distance from the site of the cartilage defect (Figure 6c). The histology of these lumps is shown in Figure $7 \mathrm{~b}$ and corresponds to a disordered growth of cartilaginous tissue. Group $M$ had a very irregular surface in $75 \%$ of the cases, having generally a fibrous appearance, showing a repaired surface with a matt appearance, bulges, and filiform projections and occasionally fissures between native and repaired tissues.

Subchondral cysts present in the PLLA and $\mathrm{CHT}$ group were present in $44.4 \%$ of the PLLA+CHT samples, at a main depth of 
FIGURE 6 Macroscopic visualization of the regenerated cartilage, (a) and (b), frontal and cross-sectional views after implantation of PLLA microspheres covered by the membrane, (c) implantation of PLLA and chitosan microspheres, and (d) only microfracture of subchondral bone covered by the membrane. Arrowheads point some of the superficial lumps. PLLA, poly(L-lactic acid)

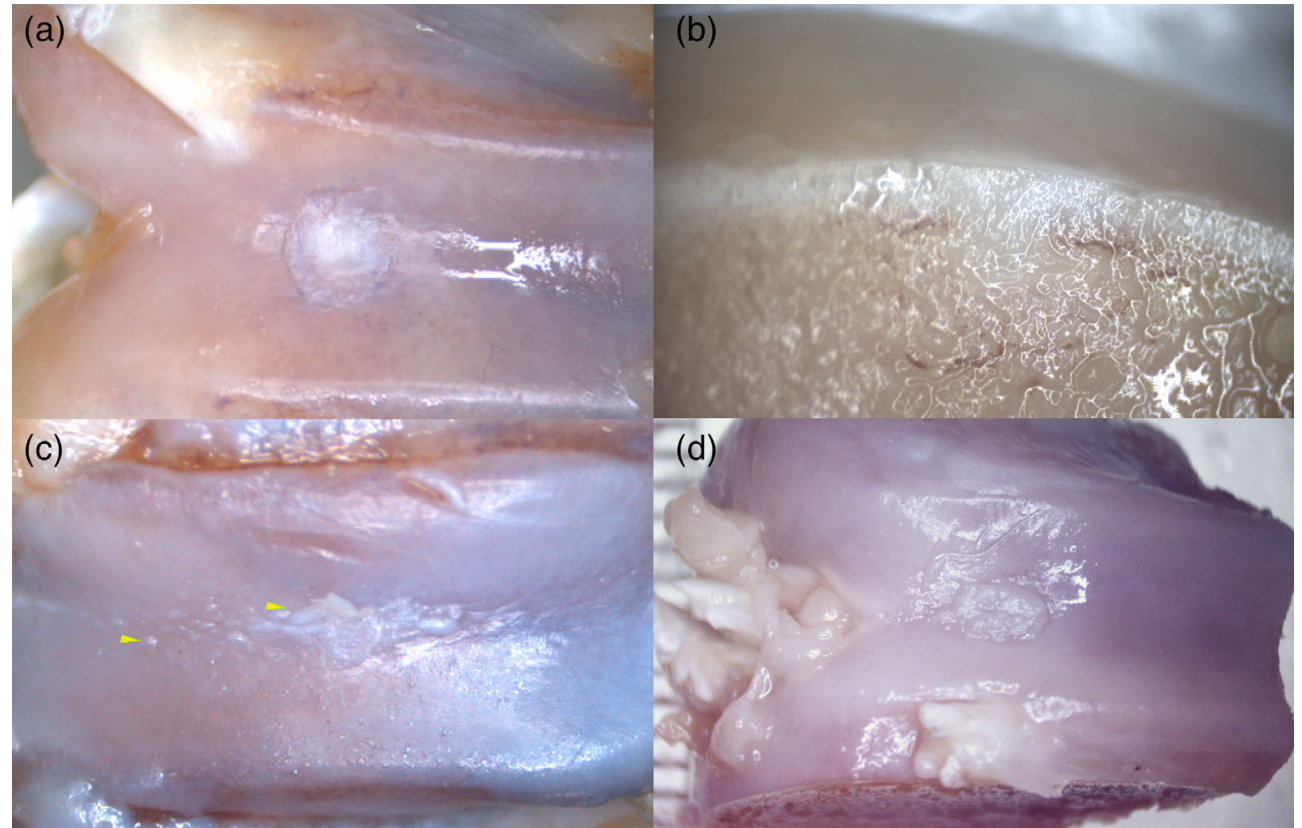

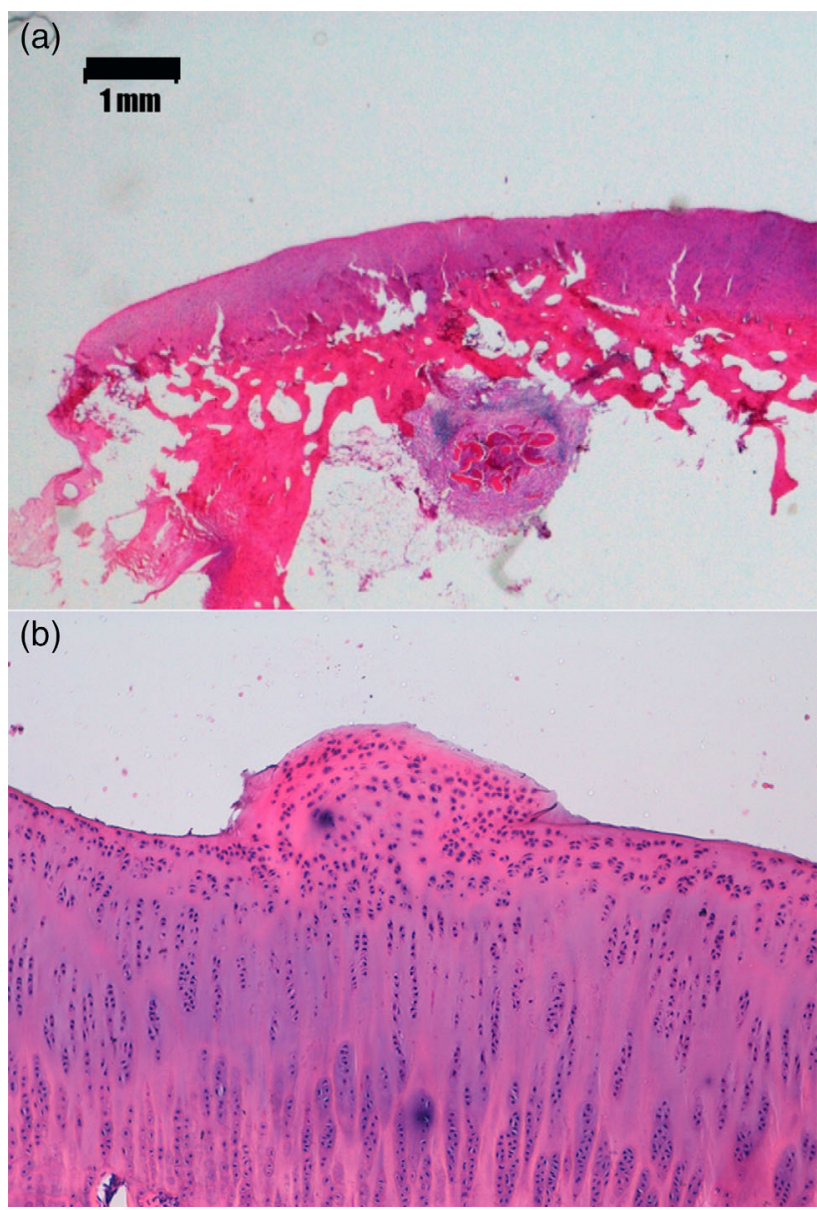

FIGURE 7 Histological views (H\&E) showing (a) the formation of cysts and (b) lumps in the articular surface in poly(L-lactic acid) + chitosan (PLLA+CHT) series. H\&E, hematoxylin-eosin
$2,421 \pm 979 \mu \mathrm{m}$ from the surface, and had a mean area of $3.6 \pm 0.96 \times 10^{6} \mu \mathrm{m}^{2}$.

\section{4 | DISCUSSION}

There are currently many research studies on the use of microspheres in regenerative medicine and delivery drugs. However, little is known about the influence of the nature of polymers commonly used to prepare microspheres on their interaction with macrophages and on the induced release of inflammation mediators (Bitencourt Cda et al., 2015). Macrophages are used in a very sensitive culture model to evaluate possible in vitro inflammatory responses to a material with potential application in regenerative medicine (Denlinger et al., 1996; Fernández et al., 2014; Lastra et al., 2017; Lastra et al., 2018).

Several researchers have also reported good results on the cell viability of osteoblasts (Zan, Wang, Dong, Cheng, \& Tian, 2008), chondrocytes (Lao et al., 2008), and stromal cells (García Cruz et al., 2008) cultured in the presence of microspheres, similarly to the present study. In addition, Zan et al. (2008) did not find significant differences in cell viability when the cells grow with $\mathrm{CHT}$ microspheres even after 10 days of culture.

Luzardo-Alvarez et al. (2005) also studied the NO production for RAW264.7 macrophages with CHT microspheres. They found that $\mathrm{CHT}$ did not induce significant levels of NO production as compared with control conditions, but when the cells were stimulated with lipopolysaccharide (LPS), the produced levels of NO were six times higher than those of the control. They also evaluated the effect of $\mathrm{CHT}$ microspheres to macrophages on tumor necrosis factor alpha (TNF- $\alpha$ ) production and found that this cytokine was unaffected by the treatment. 
Overall, our results suggest that the microspheres do not induce cytotoxicity effects on macrophages for the duration tested. Macrophages are the first line of defense and recognition of foreign substances and microorganisms (Gordon, 2007), hence the importance of our results. This hypothesis, however, was later confirmed using the in vivo model.

A rabbit knee model has been widely used as an in vivo model for articular cartilage regeneration (Bonasia et al., 2016; Dashtdar et al., 2015). Our group has previously studied the regeneration of articular cartilage in a rabbit knee model using the combination of a macroporous implant and the stimulation of the subchondral bone by microfracture (Lebourg et al., 2014; Martinez-Diaz et al., 2010; Sancho-Tello et al., 2015; Sancho-Tello et al., 2017). The results pointed to the displacement of the implanted biomaterial out of the area of regeneration penetrating the subchondral bone, allowing a tissue with the histological characteristics of the hyaline cartilage to form between the scaffold and the articular surface. This is a very promising result because, on the one hand, it allows the organization of the newly formed tissue without the impediment of the presence of the scaffolding material and, on the other hand, the degradation time of the biomaterial needs not to be adjusted (can be longer) to the regenerated cartilage formation. In this study, we intend to continue in this line, making it easier for the new tissue to move the supporting biomaterial and acquire the proper organization of the hyaline cartilage.

Particles' displacement is more effective in this case. For sure, PLLA microspheres are not reabsorbed, but there are no proofs about degradation of $\mathrm{CHT}$ microspheres that could be inserted into the cysts (as we will see below) formed in the subchondral cancellous bone area. The shift of the biomaterial out of the cartilage regeneration zone allows the organization of the newly formed cartilage with the characteristic ordering of hyaline cartilage, with chondrocytes isolated in lacunae and aligned in columns perpendicular to the articular surface. In this way, articular surface has a smooth appearance in the macroscopic view (Figure 6). Microspheres displacement was found in all the cases with a single exception in one case when only PLLA microspheres were implanted, and PLLA remaining microspheres appeared into the newly formed cartilage.

Dynamic compression in the site of the regeneration should play an important role in the remodeling of the regeneration site along time. In a previous work (Sancho-Tello et al., 2015), we followed the invasion of the cartilage defect along time in a rabbit knee model in which a scaffold was implanted in the cartilage defect after injuring subchondral bone. Just 1 week after implantation, a layer of cells and extracellular matrix was formed at the articular surface attached to the scaffold external surface, and this layer became thicker as regeneration time increased. At the same time, the scaffold pores were invaded by cells. In the present study, we have data only for the regeneration time of 3 months, but the fact that the membrane that covers the defect is pushed down toward subchondral bone suggests the initial formation of a layer of tissue on top of the practiced cartilage defect.

A comparison of the quality of the regenerated cartilage after implantation of PLLA, PLLA+CHT, or no microspheres is performed based on macroscopic and microscopic evaluations. Since two exitus occurred, the lack of new animals that would qualify for the methodology used within the established timeframes justifies the difference in group sizes (7 in group PLLA vs 9 in group PLLA+CHT).

\section{1 | Macroscopic evaluation}

Individual scores for each category and group are summed up in Table 1. PLLA series obtained the highest mean score (10.59 \pm 0.79$)$, PLLA+CHT obtained a slightly lower mean score (9.38 \pm 1.51 ), group $M$ had the lowest mean score $(8.50 \pm 1.29)$, and control group obtained the maximum scores (12) as it was expected. Significant differences were observed in PLLA series $(p=.018)$ for the macroscopic appearance category, resulting in better scores.

All intraobserver kappa correlation index $(\kappa)$ for macroscopic evaluation were found to be "moderate" or higher, with $62.5 \%$ of the results having a correlation of "substantial" or "almost perfect." Interobserver $\kappa$ values were found to be "fair" or "moderate."

Significant differences were observed in the category macroscopic surface assessment, with higher results in PLLA series (Table 1). This implies a more regular surface, with the absence of fissures or fibrillations. All of the experimental groups' total numbers were in the range 8-11, thus being described as "nearly normal" regeneration cartilage by the ICRS scale. However, PLLA series was in the upper limit, while both groups PLLA+CHT and $\mathrm{M}$ obtained total numbers that placed them in the lower limit of the grade. This is due to sufficient thickness and integration of the repaired or regenerated tissue in all groups and does not imply a hyaline-like cartilage.

\subsection{Microscopic evaluation}

Histological parameters are listed in Table 2. In all of the experimental groups, a small invagination was observed where the reparative/regenerative area and the native cartilage met. An increase in the vascularization of the subchondral bone was also noted in comparison

TAB LE 1 ICRS macroscopic evaluation of cartilage repair

\begin{tabular}{|llll|}
\hline Category & PLLA & PLLA+CHT & Membrane (M) \\
\hline $\begin{array}{c}\text { Degree of } \\
\text { defect repair }\end{array}$ & $3.86 \pm 0.38$ & $3.25 \pm 0.89$ & $3.25 \pm 0.96$ \\
$\begin{array}{c}\text { Integration to } \\
\text { border zone }\end{array}$ & $3.00 \pm 0.00$ & $3.38 \pm 0.52$ & $2.75 \pm 0.50$ \\
\hline $\begin{array}{l}\text { Macroscopic } \\
\text { appearance }\end{array}$ & $3.71 \pm 0.76 *$ & $2.75 \pm 0.46$ & $2.50 \pm 1.29$ \\
\hline Total & $10.57 \pm 0.79$ & $9.38 \pm 1.51$ & $8.50 \pm 1.29$ \\
\hline Grade & II & II & II \\
\hline
\end{tabular}

Notes: Data are represented as means $\pm S D$. Statistical significance was determined by $\chi^{2}$ test, and $p<.05$ was considered statistically significant. Results for native cartilage are not presented, as they were always the maximum (4 in each category, with a total of 12), as to be expected from hyaline cartilage.

Abbreviations: CHT, chitosan; ICRS, International Cartilage Repair Society; PLLA, poly(L-lactic acid).

$* p<0.05$. 
TABLE 2 ICRS II scale

\begin{tabular}{|l|ccc|}
\hline Histological parameter & PLLA & PLLA+CHT & Membrane \\
\hline Tissue morphology & $75.0 \pm 14.4$ & $86.1 \pm 18.2$ & $25.0 \pm 28.9 *$ \\
\hline Matrix staining & $53.6 \pm 33.6 *$ & $88.9 \pm 13.2$ & $18.8 \pm 12$. * $^{*}$ \\
\hline Cell morphology & $78.6 \pm 30.4$ & $94.4 \pm 9.4$ & $31.3 \pm 31.5$ \\
\hline Chondrocyte clustering & $75.0 \pm 14.4$ & $75.0 \pm 37.5$ & $50.0 \pm 45.6$ \\
\hline Surface architecture & $96.4 \pm 9.4$ & $86.1 \pm 18.2$ & $25.0 \pm 35.4 *$ \\
\hline Basal integration & $67.9 \pm 37.4$ & $77.8 \pm 26.4$ & $62.5 \pm 43.3$ \\
\hline Marrow fibrosis & $100.0 \pm 0.0$ & $86.1 \pm 33.3$ & $93.8 \pm 12.5$ \\
\hline Inflammation & $100.0 \pm 0.0$ & $97.2 \pm 8.3$ & $75.0 \pm 50.0$ \\
\hline Abnormal calcification & $100.0 \pm 0.0$ & $100.0 \pm 0.0$ & $100.0 \pm 0.0$ \\
\hline Vascularization & $96.4 \pm 9.4$ & $100.0 \pm 0.0$ & $100.0 \pm 0.0$ \\
\hline Surface assessment & $82.1 \pm 18.9$ & $83.3 \pm 12.5$ & $12.5 \pm 14.4 *$ \\
\hline Mid/deep zone assessment & $78.6 \pm 17.3$ & $72.2 \pm 31.7$ & $31.3 \pm 37.5$ \\
\hline Overall assessment & $78.6 \pm 17.3$ & $80.6 \pm 20.8$ & $31.3 \pm 37.5$ \\
\hline Mean ICRS II & $83.2 \pm 9.7$ & $86.8 \pm 10.6$ & $50.5 \pm 20.6$ \\
\hline
\end{tabular}

Notes: Each parameter is scored using a 100-unit visual analogue scale, with a score of 0 being assigned for properties considered indicative of fibrous cartilage or poor quality articular hyaline cartilage, and 100 for good quality articular hyaline cartilage, and are presented as mean $\pm S D$. Statistical significance was determined by $\chi^{2}$ test, and $p<.05$ was considered statistically significant. Results for the control group are not presented, as they were always the maximum (100), as to be expected from hyaline cartilage. Tidemark values are not included as they could not be measured. Mean ICRS II values were calculated with the other 13 parameters.

Abbreviations: $\mathrm{CHT}$, chitosan; ICRS, International Cartilage Repair Society; PLLA, poly(L-lactic acid).

$* p<0.05$.

with that of native cartilage. Whenever there were different qualities of cartilage regeneration obtained, seriated samples were taken and the scores were calculated with those considered to be more representative.

PLLA series had a slightly thicker cartilage, with better tissue architecture in the areas nearest to the native cartilage. Its cell morphology was normal, with chondrocytes having a basophilic cytoplasm and no apparent morphological changes. Chondrocyte clustering was generally observed in both the peripheral areas of regenerated cartilage and the adjacent native areas (Figure 5a).

In the PLLA+CHT group, normal cellularity was also observed, with a slightly thicker regeneration cartilage and the presence of clustering, besides the appearance of lumps aforementioned. It is worth noting that the subchondral cysts are formed in $66.7 \%$ of the cases (Figure 7a), with multinucleated cells and remains of membranes and biomaterials within them. These cysts appear quite far from the articular surface deep in the subchondral spongy bone tissue, which might weaken the joint. The series $M$ obtained poor cartilage regeneration, similar to a fibrous repair. Cartilage thickness was variable, with cases of increases or decreases with respect to native ones. The tissue obtained was poorly organized and the surface was found to be generally irregular (Figure $5 \mathrm{~d}$ ).

All interobserver $\kappa$ values for macroscopic evaluation were found to be "fair" or higher, with $68.5 \%$ of the results having a correlation of "substantial" or "almost perfect."
Significant differences were observed in the categories such as tissue morphology, surface architecture, and superficial assessment. In them, group $M$ had significantly lower scores compared with groups PLLA or PLLA+CHT. No significant differences were found between PLLA and PLLA+CHT series and native cartilage (control group), suggesting a high-quality regeneration cartilage.

The categories added to the ICRS II score to evaluate material reabsorption had a significant difference in the presence of PLLA microspheres. However, the presence of subchondral cysts was only observed in PLLA+CHT. A possible explanation would be that biomaterials were reabsorbed at a similar rate, but whereas PLLA was found as microspheres, $\mathrm{CHT}$ would have been dragged into these cysts in the subchondral bone, probably originating from an immune response already described in the literature with biomaterials (Chu et al., 2016; Conoscenti et al., 2017), although our in vitro experiments only showed an increase in NO production in the PLLA+CHT group after culturing for $24 \mathrm{hr}$.

It must be noted that the time of evolution of the regeneration prior to the sacrifice of the rabbits was 12 weeks. This time was decided as it is within the range established by the ICRS (Zhu et al., 2014), and longer times do not seem to improve the quality of cartilage regeneration (Kumar, Muzzarelli, Muzzarelli, Sashiwa, \& Domb, 2004; Teté et al., 2007). Nevertheless, it is shorter than the half-life of both PLLA and CHT, so the presence of the biomaterial in the samples was expected. Further studies with different evolution times could clarify the matter.

Differences were also found in surface architecture and surface assessment, having significantly worse results in group $M$. This was further demonstrated by significantly better values of interdigitation index in groups PLLA, PLLA+CHT, and control compared with group $M$, while no differences were observed when comparing groups PLLA and PLLA+CHT with the control group. Hence, microspheres scaffolding with PLLA and CHT result in a tissue whose surface is both microscopically and numerically equivalent to normal hyaline cartilage.

\section{3 | Morphometric evaluation}

Individual morphometric values for each category and group are summarized up in Table 3. Some parameters lack clear reference values in the literature, and hence must be approached with care. Cell density increases both in immature cartilage and in fibrocartilage, an increase observed in the $M$ series with respect to the native cartilage was expected, but the increase observed in groups PLLA+CHT and $M$ and the decrease observed in group PLLA have unclear meaning. Chondrocyte clustering was also described as a negative feature associated with degeneration in ostheoarthritic tissue (Vikingsson et al., 2015). Other studies interpret these clusters as a sign of immature cartilage during the healing process (Bell, Hurtig, Quenneville, Rivard, \& Hoemann, 2017; Kumar et al., 2004; Kuo et al., 2011). Regenerated tissues were generally thicker than native cartilage, as was the subchondral bone beneath them. Interdigitation index showed 


\begin{tabular}{|lcccc|}
\hline Morphometry parameter & PLLA & PLLA+CHT & Membrane & Native \\
\hline Cartilage thickness $(\mu \mathrm{m})$ & $705 \pm 292$ & $711 \pm 215$ & $670 \pm 244$ & $478 \pm 94$ \\
\hline $\begin{array}{l}\text { Subchondral bone } \\
\text { thickness }(\mu \mathrm{m})\end{array}$ & $786 \pm 195$ & $720 \pm 225$ & $566 \pm 193$ & $650 \pm 235$ \\
\hline Cell density $\left(\mathrm{cell} s / \mathrm{mm}^{2}\right)$ & $1,498 \pm 149$ & $2,366 \pm 1,041$ & $2,579 \pm 1,490$ & $1914 \pm 466$ \\
\hline Interdigitation index & $1.14 \pm 0.10$ & $1.28 \pm 0.14$ & $1.73 \pm 0.60 *$ & $1.03 \pm 0.02$ \\
\hline
\end{tabular}

TABLE 3 Morphometrical

parameters measured

Abbreviations: CHT, chitosan; ICRS, International Cartilage Repair Society; PLLA, poly(L-lactic acid).

$* p<.05$.

significantly lower values in the control group, with no differences observed in groups PLLA, PLLA+CHT, and $\mathrm{M}$.

\section{5 | CONCLUSIONS}

The regeneration of the articular cartilage requires both the presence of cells with chondrogenic capacity in the site of the defect and a mechanical support capable of transmitting to the cells the dynamic compression efforts to which the cartilage is subjected in vivo, so as to stimulate the characteristic organization of the hyaline cartilage. In the regeneration strategy presented in this article, mesenchymal stem cells come from the microfracture of subchondral bone, while the mechanical support role is played by the microspheres, either PLLA or a mixture of PLLA and CHT. In both cases, the histological characteristics of the regenerated tissue are those of the hyaline cartilage that can grow with its characteristic arrangement, thanks to the simultaneous displacement of the implanted microspheres toward the subchondral bone. Interestingly enough, even the membrane that was placed covering the microspheres at the time of the intervention is also displaced toward subchondral bone. The system is very promising as a strategy of regeneration; however, the presence of $\mathrm{CHT}$ has produced certain anomalous effects, such as the formation of lumps on the articular surface outside the region where the defect was created, and that has the structure of a cartilaginous disordered tissue, and also the formation of cysts in the bone at a considerable distance from the articular surface. It is noteworthy that neither the $\mathrm{CHT}$ nor the PLLA microspheres showed any inflammatory response that could be highlighted in the trials carried out on cultures with macrophages.

\section{ACKNOWLEDGMENTS}

Financial support from the Spanish Ministry of Economy and Competitiveness (MINECO) through the project MAT2016-76039-C4-(1 and 2)-R (Agencia Estatal de Investigación/Fondo Europeo de Desarrollo Regional de la Unión Europea) is acknowledged (Spanish group). This work was also partially supported by grants from Comisión de Investigaciones Científicas de la Provincia de Buenos Aires (CICPBA), Universidad Nacional de La Plata, Proyecto 11/X643 (Argentina group). The consorcio Centro de Investigación Biomédica en Red, Bioingeniería, Biomateriales y Nanomedicina, CIBER-BBN is an initiative funded by the VI National R\&D\&I Plan 2008-2011, Iniciativa Ingenio 2010, Consolider Program. CIBER Actions are financed by the Instituto de Salud Carlos III with assistance from the European Regional Development Fund.
M.L.L. is a Doctoral Fellow of Universidad Nacional de La Plata, J.M.F. is Investigador Adjunto of the Consejo Nacional de Investigaciones Científicas y Técnicas, CONICET, and A.M.C. is Investigador Principal de la Comisión de Investigaciones Científicas de la Provincia de Buenos Aires, CICPBA.

\section{REFERENCES}

Allepuz, A., Martínez, O., Tebé, C., Nardi, J., Portabella, F., \& Espallargues, M. (2014). Joint registries as continuous surveillance systems: The experience of the catalan arthroplasty register (RACat). The Journal of Arthroplasty, 29, 484-490.

Almeida, C. R., Serra, T., Oliveira, M. I., Planell, J. A., Barbosa, M. A., \& Navarro, M. (2014). Impact of 3-D printed PLA- and chitosan-based scaffolds on human monocyte/macrophage responses: Unraveling the effect of 3-D structures on inflammation. Acta Biomaterialia, 10(2), 613-622.

Bell, A. D., Hurtig, M. B., Quenneville, E., Rivard, G.-É., \& Hoemann, C. D. (2017). Effect of a rapidly degrading presolidified $10 \mathrm{kDa}$ chitosan/ blood implant and subchondral marrow stimulation surgical approach on cartilage resurfacing in a sheep model. Cartilage, 8(4), 417-431.

Bitencourt Cda, S., Silva, L. B., Pereira, P. A., Gelfuso, G. M., \& Faccioli, L. H. (2015). Microspheres prepared with different copolymers of poly(lactic-glycolic acid) (PLGA) or with chitosan cause distinct effects on macrophages. Colloids and Surfaces. B, Biointerfaces, 136, 678-686.

Bonasia, D. E., Martin, J. A., Marmotti, A., Kurriger, G. L., Lehman, A. D., Rossi, R., \& Amendola, A. (2016). The use of autologous adult, allogenic juvenile, and combined juvenile-adult cartilage fragments for the repair of chondral defects. Knee Surgery, Sports Traumatology, Arthroscopy, 24(12), 3988-3996.

Carmona, L., Ballina, J., \& Gabriel, R. (2001). The burden of musculoskeletal diseases in the general population of Spain: Results from a national survey. Annals of the Rheumatic Diseases, 60, 1040-1045.

Chu, J., Zeng, S., Gao, L., Groth, T., Li, Z., Kong, J., ... Li, L. (2016). Poly (Llactic acid) porous scaffold-supported alginate hydrogel with improved mechanical properties and biocompatibility. The International Journal of Artificial Organs, 39(8), 435-443.

Conoscenti, G., Schneider, T., Stoelzel, K., Carfi Pavia, F., Brucato, V., Goegele, C., ... Schulze-Tanzil, G. (2017). PLLA scaffolds produced by thermally induced phase separation (TIPS) allow human chondrocyte growth and extracellular matrix formation dependent on pore size. Materials Science and Engineering: C, 80, 449-459.

Dashtdar, H., Murali, M. R., Abbas, A. A., Suhaeb, A. M., Selvaratnam, L., Tay, L. X., \& Kamarul, T. (2015). PVA-chitosan composite hydrogel versus alginate beads as a potential mesenchymal stem cell carrier for the treatment of focal cartilage defects. Knee Surgery, Sports Traumatology, Arthroscopy, 23(5), 1368-1377.

Denlinger, L. C., Fisette, P. L., Garis, K. A., Kwon, G., Vazquez-Torres, A., Simon, A. D., ... Corbett, J. A. (1996). Regulation of inducible nitric oxide synthase expression by macrophage purinoreceptors and calcium. The Journal of Biological Chemistry, 271, 337-342. 
Fernández, J. M., Cortizo, M. S., \& Cortizo, A. M. (2014). Fumarate/ceramic composite based scaffolds for tissue engineering: Evaluation of hydrophylicity, degradability, toxicity and biocompatibility. J Biomater Tissue Eng, 4(3), 227-234.

García Cruz, D. M., Escobar Ivirico, J. L., Gomes, M. M., Gómez Ribelles, J. L., Salmerón Sánchez, M., Reis, R. L., \& Mano, J. F. (2008). Chitosan microparticles as injectable scaffolds for tissue engineering. Tissue Eng Regen Med, 2(6), 378-380.

Gordon, S. (2007). The macrophage: Past, present and future. European Journal of Immunology, 37, S9-S17.

Goyal, D., Keyhani, S., Lee, E. H., \& Hui, J. H. (2013). Evidence-based status of microfracture technique: A systematic review of level I and II studies. Arthroscopy, 29, 1579-1588.

Hangody, L., Kish, G., Kárpáti, Z., Udvarhelyi, I., Szigeti, I., \& Bély, M. (1998). Mosaicplasty for the treatment of articular cartilage defects: Application in clinical practice. Orthopedics, 21, 751-756.

Hoemann, C., Kandel, R., Roberts, S., Saris, D. B. F., Creemers, L., MainilVarlet, P., ... Buschmann, M. D. (2011). International Cartilage Repair Society (ICRS) recommended guidelines for histological endpoints for cartilage repair studies in animal models and clinical trials. Cartilage, 2(2), 153-172.

Kumar, M. N. V. R., Muzzarelli, R. A. A., Muzzarelli, C., Sashiwa, H., \& Domb, A. J. (2004). Chitosan chemistry and pharmaceutical perspectives. Chemical Reviews, 104(12), 6017-6084.

Kuo, T. F., Lin, M. F., Lin, Y. H., Lin, Y. C., Su, R. J., Lin, H. W., \& Chan, W. P. (2011). Implantation of platelet-rich fibrin and cartilage granules facilitates cartilage repair in the injured rabbit knee: Preliminary report. Clinics, 66(10), 1835-1838.

Landis, J. R., \& Koch, G. G. (1977). The measurement of observer agreement for categorical data. Biometrics, 33(1), 159.

Lao, L., Tan, H., Wang, Y., \& Gao, C. (2008). Chitosan modified poly (Llactide) microspheres as cell microcarriers for cartilage tissue engineering. Colloids and Surfaces. B, Biointerfaces, 66(2), 218-225.

Lastra, M. L., Molinuevo, M. S., Blaszczyk-Lezak, I., Mijangos, C., \& Cortizo, M. S. (2018). Nanostructured fumarate copolymer-chitosan crosslinked scaffold: An in vitro osteochondrogenesis regeneration study. Journal of Biomedical Materials Research. Part A, 106(2), 570-579.

Lastra, M. L., Molinuevo, M. S., Cortizo, A. M., \& Cortizo, M. S. (2017). Fumarate copolymer-chitosan cross-linked scaffold directed to osteochondrogenic tissue engineering. Macromolecular Bioscience, 17(5), 1600219.

Lebourg, M., Martínez-Díaz, S., García-Giralt, N., Torres-Claramunt, R., Gómez-Tejedor, J. A., Ribelles, J. L., ... Monllau, J. C. (2014). Cell-free cartilage engineering approach using hyaluronic acid-polycaprolactone scaffolds: A study in vivo. Journal of Biomaterials Applications, 28(9), 1304-1315.

Luzardo-Alvarez, A., Blarer, N., Peter, K., Romero, J. F., Reymond, C., Corradin, G., \& Gander, B. (2005). Biodegradable microspheres alone do not stimulate murine macrophages in vitro, but prolong antigen presentation by macrophages in vitro and stimulate a solid immune response in mice. Journal of Controlled Release, 109(1-3), 62-76.

Mainil-Varlet, P., Van Damme, B., Nesic, D., Knutsen, G., Kandel, R., \& Roberts, S. (2010). A new histology scoring system for the assessment of the quality of human cartilage repair: ICRS II. The American Journal of Sports Medicine, 38(5), 880-890.
Martinez-Diaz, S., Garcia-Giralt, N., Lebourg, M., Gómez-Tejedor, J. A., Vila, G., Caceres, E., ... Monllau, J. C. (2010). In vivo evaluation of 3-dimensional polycaprolactone scaffolds for cartilage repair in rabbits. The American Journal of Sports Medicine, 38(3), 509-519.

McCormick, F., Harris, J. D., Abrams, G. D., Frank, R., Gupta, A., Hussey, K., ... Cole, B. (2014). Trends in the surgical treatment of articular cartilage lesions in the United States: An analysis of a large private-payer database over a period of 8 years. Arthroscopy, 30, 222-226.

Sancho-Tello, M., Forriol, F., Gastaldi, P., Ruiz-Saurí, A., Martín De Llano, J. J., Novella-Maestre, E., ... Carda, C. (2015). Time evolution of in vivo articular cartilage repair induced by bone marrow stimulation and scaffold implantation in rabbits. The International Journal of Artificial Organs, 38(4), 210-223.

Sancho-Tello, M., Forriol, F., Martín de Llano, J. J., Antolinos-Turpin, C., Gómez-Tejedor, J. A., Gómez Ribelles, J. L., \& Carda, C. (2017). Biostable scaffolds of polyacrylate polymers implanted in the articular cartilage induce hyaline-like cartilage regeneration in rabbits. The International Journal of Artificial Organs, 40(7), 350-357.

Steadman, J. R., Rodkey, W. G., Briggs, K. K., \& Rodrigo, J. J. (1999). The microfracture technic in the management of complete cartilage defects in the knee joint. Orthopade, 28, 26-32.

Teté, S., Mastrangelo, F., Carone, L., Nargi, E., Costanzo, G., Vinci, R., ... Ciccarelli, R. (2007). Morphostructural analysis of human follicular stem cells on highly porous bone hydroxyapatite scaffold. International Journal of Immunopathology and Pharmacology, 20(4), 819-826.

Van den Borne, M. P. J., Raijmakers, N. J. H., Vanlauwe, J., Victor, J., de Jong, S. N., Bellemans, J., \& Saris, D. B. (2007). International Cartilage Repair Society (ICRS) and Oswestry macroscopic cartilage evaluation scores validated for use in autologous chondrocyte implantation (ACI) and microfracture. Osteoarthritis and Cartilage, 15(12), 1397-1402.

Vikingsson, L., Sancho-Tello, M., Ruiz-Saurí, A., Martínez Díaz, S., GómezTejedor, J. A., Gallego Ferrer, G., ... Gómez Ribelles, J. L. (2015). Implantation of a polycaprolactone scaffold with subchondral bone anchoring ameliorates nodules formation and other tissue alterations. The International Journal of Artificial Organs, 38(12), 659-666.

Zan, Q., Wang, C., Dong, L., Cheng, P., \& Tian, J. (2008). Effect of surface roughness of chitosan-based microspheres on cell adhesion. Applied Surface Science, 255(2), 401-403.

Zhang, C., Cai, Y. Z., \& Lin, X. J. (2016). One-step cartilage repair technique as a next generation of cell therapy for cartilage defects: Biological characteristics, preclinical application, surgical techniques, and clinical developments. Arthroscopy, 32, 1444-1450.

Zhu, W., Chen, K., Lu, W., Sun, Q., Peng, L., Fen, W., ... Zeng, Y. (2014). In vitro study of nano-HA/PLLA composite scaffold for rabbit BMSC differentiation under TGF- $\beta 1$ induction. Vitr Cell Dev Biol - Anim, 50(3), 214-220.

How to cite this article: Zurriaga Carda J, Lastra ML, Antolinos-Turpin CM, et al. A cell-free approach with a supporting biomaterial in the form of dispersed microspheres induces hyaline cartilage formation in a rabbit knee model. J Biomed Mater Res. 2019;1-11. https://doi.org/10.1002/jbm. b.34490 